\title{
Downregulation of phosphoglycerate mutase 5 improves microglial inflammasome activation after traumatic brain injury
}

\author{
Yuhua Chen ${ }^{1,2,3}$, Kai Gong $\mathbb{D}^{2,4}$, Limin Guo ${ }^{2,3}$, Bingchang Zhang ${ }^{2,4}$, Sifang Chen ${ }^{2,4}$, Zhangyu Li ${ }^{2,4}$, Xu Quanhua $^{5}$, Wei Liu (D) ${ }^{1,2,4}{ }^{凶}$ and \\ Zhanxiang Wang (iD) $2,4 \times$
}

(c) The Author(s) 2021

\begin{abstract}
Traumatic brain injury (TBI) is considered as the most common cause of disability and death, and therefore an effective intervention of cascade pathology of secondary brain injury promptly can be a potential therapeutic direction for TBI prognosis. Further study of the physiological mechanism of TBI is urgent and important. Phosphoglycerate mutase 5 (Pgam5), a mitochondrial protein, mediate mitochondrial homeostasis, cellular senescence, and necroptosis. This study evaluated the effects of Pgam5 on neurological deficits and neuroinflammation of controlled cortical impact-induced TBI mouse model in vivo and LPS + ATP-induced microglia model in vitro. Pgam5 was overexpressed post-TBI. Pgam5 depletion reduced pyroptosis-related molecules and improved microglia activation, neuron damage, tissue lesion, and neurological dysfunctions in TBI mice. RNA-seq analysis and molecular biology experiments demonstrated that Pgam5 might regulate inflammatory responses by affecting the post-translational modification and protein expression of related genes, including NIrp3, caspase1, Gsdmd, and II-1 $\beta$. In microglia, Pgam5-sh abrogated LPS + ATPinduced II-1 $\beta$ secretion through Asc oligomerization-mediated caspase-1 activation, which was independent of Rip3. The data demonstrate the critical role Pgam5 plays in nerve injury in the progression of TBI, which regulates Asc polymerization and subsequently caspase 1 activation, and thus reveals a fundamental mechanism linking microglial inflammasome activation to Asc/ caspase1-generated II-1 $\beta$-mediated neuroinflammation. Thus, our data indicate Pgam5 worsens physiological and neurological outcomes post-TBI, which may be a potential therapeutic target to improve neuroinflammation after TBI.
\end{abstract}

Cell Death Discovery (2021)7:290; https://doi.org/10.1038/s41420-021-00686-8

\section{BACKGROUND}

Traumatic brain injury (TBI), a global health challenge, is known to be one of the most common causes of death and disability in young men, and it imposes a burden on patients and their families, as well as a society [1, 2]. Typically, a complex set of pathophysiological processes leads to severe secondary brain injury within hours or days of acute brain injury, which follows the primary injury-mediated neuroinflammation, immune response, oxidative stress, mitochondrial dysfunction, and apoptosis [3, 4]. Neuroinflammation is believed to play an important role in secondary changes $[4,5]$. Because in primary brain injury, the opportunity of treatment can be lost within a very short period, the effective intervention of cascade pathology of secondary brain injury promptly will become a potential therapeutic direction for TBI [6]. Thus, it is urgent and important to further study the physiological mechanism of neuroinflammation and nerve injury to alleviate the poor prognosis of TBI patients.

Phosphoglycerate mutase 5 (Pgam5), a mitochondrial protein, localized to the mitochondrial outer-inner membrane contact sites, its activation leads to a linear fracture of string-arranged mitochondria, and this phenomenon mediated mitochondrial and cellular fate [7], including cellular senescence [8] and early stages of necroptosis [9-11]. Some studies have suggested that Pgam5 drives necroptosis through imposing mitochondrial quality control via Drp1 phosphorylation $[12,13]$. Pgam5 plays an important role in cardiac microvascular and skin ischemia-reperfusion injury through the Pgam5/CypD/mPTP pathway and Pgam5/Drp1 necrotic pathway $[14,15]$. Previously, we demonstrated Pgam5 involved in the progression of neuronal injury following TBI via Drp1 activation-mediated mitochondrial dysfunction [16]. Furthermore, Pgam5 involves regulating $\mathrm{II}-1 \beta$ secretion in mouse bone marrow-derived dendritic cells (BMDCs) [10, 17]. However, whether Pgam5 is involved in II-1 $\beta$ secretion and neuroinflammation post-TBI and by what pathway is still unclear.

Pyroptosis, a kind of inflammatory cell necrosis, is mainly characterized by the activation of caspase 1 and the secretion of mature II-1 $\beta$ and II-18 and where NIrp3 inflammasome acts as the pivotal regulatory signal of pyroptosis, which serves as a potential biomarker and therapeutic target [18]. In our previous studies, we found TBI was accompanied by increased neuroinflammation and pyroptosis during the acute phase post-TBI [19], which were significantly relieved by suppressing Nlrp3 inflammasomemediated pyroptosis [19-21]. Thus, exploring the regulation mechanism of the NLRP3 inflammasome may provide a new idea

\footnotetext{
${ }^{1}$ Department of Central Laboratory, Xi'an Peihua University, Xi'an 710125, China. ${ }^{2}$ Trauma Center, First Affiliated Hospital of Xiamen University, 55 Zhenhai Rd, Xiamen 361003 Fujian, China. ${ }^{3}$ Department of Anatomy and Neurobiology, Central South University, 172 Tongzipo Rd, Changsha 410013 Hunan, China. ${ }^{4}$ Department of Neurosurgery, Xiamen Key Laboratory of Brain Center, The First Affiliated Hospital of Xiamen University, 55 Zhenhai Rd, Xiamen 361003 Fujian, China. ${ }^{5}$ Department of Neurosurgery, Bijie Traditional Chinese Medical Hospital, Bijie 551700, China. ${ }^{凶}$ email: vincelau@sina.com; wangzx@xmu.edu.cn
}

Received: 14 March 2021 Revised: 20 September 2021 Accepted: 29 September 2021

Published online: 12 October 2021 
for regulating the secretion of inflammatory factor $I I-1 \beta$ and neuroinflammation after TBI.

This study aims to explore the potential role and molecular mechanism of Pgam5 in the pathogenesis of TBI. Pgam5 depletion was used in vivo controlled cortical impact (CCI) TBI mouse model and in vitro lipopolysaccharide (LPS)-induced microglia models to demonstrate the role of Pgam5 in downstream neuroinflammation post-TBI. Our data indicate that Pgam5 facilitates physiological processes of $\mathrm{TBI}$, which may be presented as a promising therapeutic target post-TBI.

\section{MATERIALS AND METHODS \\ CCI model}

We carried out our experiments on Pgam5-deficient (Pgam5 ${ }^{-1-}$; Cyagen Biosciences Inc., Guangzhou, China; C57BL/6 background) adult male mice (8-10 weeks) and age-matched wild type (WT) C57BL/6 male mice. These animal experiments were approved by the Animal Care and Use Committee of First Affiliated Hospital of Xiamen University, China. A schematic diagram of the experimental design in vivo was shown in Fig. S1. Pgam5 $5^{-1-}$ and WT mice were numbered by computer and randomly divided into TBI and sham groups. About 60 mice were used in each group.

The CCI device was used to establish a TBI mice model in vivo [20]. Briefly, a $0.5 \mathrm{~mm}$ diameter hole was drilled in the right parietal cortex, $2 \mathrm{~mm}$ posterior to the bregma and $2 \mathrm{~mm}$ lateral to the sagittal suture. The $\mathrm{CCl}$ device (PinPoint ${ }^{T M}$ PCI3000; Hatteras Instruments Inc., Cary, North Carolina, USA) was adjusted to a common parameter (velocity: $5.0 \mathrm{~m} / \mathrm{s}$; depth: $2 \mathrm{~mm}$; dwell time: $100 \mathrm{~ms}$ ). The sham group underwent normal surgical procedures and not CCl. After CCl treatment, the skull was sealed and the incision was sutured. The mice were placed on a heating pad until they regained consciousness and recovered gross locomotor function. After that, the mice were put back into normal feeding units and monitored.

\section{Neurobehavioral analysis}

Neurobehavioral training and evaluation procedures were carried out according to a previous study [19]. Experimenters were unaware of the TBI or sham treatments in WT and Pgam5 ${ }^{-1-}$ mice. The mNSS, Rotarod, and open field tests were performed to evaluate neurological deficits by double-blind experiment. Before the experiments, all Pgam5 ${ }^{-1-}$ and WT mice underwent behavioral experiments and were behaved as expected for a healthy animal.

\section{Analysis of cerebral edema}

As described in previous studies $[19,21]$, brain water content was measured in 3-mm coronal sections of the ipsilateral cortex, centered upon the impact site. Tissues were immediately weighed (wet weight), then dehydrated at $100^{\circ} \mathrm{C}$ for $24 \mathrm{~h}$ to obtain the dry weight. The water content of the brain was calculated using the following formula: [(wet weight-dry weight)/wet weight] $\times 100$.

\section{Magnetic resonance Imaging (MRI) scanning}

At $48 \mathrm{~h}$ post-TBI, the mice were anesthetized by the isoflurane and $\mathrm{T2}$ weighted images were acquired using a 9.4 T small animal PharmaScan 94/ $20 \mathrm{MRI}$ scanner (Bruker BioSpin, Ettlingen, Germany) according to the following parameters: repetition time/echo time $(\mathrm{TR} / \mathrm{TE})=3000 / 50 \mathrm{~ms}$; field of view $20 \times 20 \mathrm{~mm}$, matrix $256 \times 256 \mathrm{~mm}$; coronal slice thickness $=$ $0.5 \mathrm{~mm}$; number of slices $=40$; scan time $=19 \mathrm{~min}$. The lesion was acquired based on the high-signal area of T2-weighted images.

\section{Histopathological analysis}

As described in the previous study [16], brain tissues were obtained after transcardial perfusion, and TUNEL/NeuN staining and Nissl staining were conducted. The Golgi-Cox staining was performed using the FD Rapid GolgiStain Kit following the manufacturer's instructions (FD NeuroTechnologies, Columbia, MD, USA). 6 random high-power fields were chosen and images were collected using a positive fluorescence microscope (Olympus, Osaka, Japan).

\section{RNA sequencing (RNA-seq) and data analysis}

At $48 \mathrm{~h}$ post-TBI, the samples of cortical tissue proximal to or located in the injury site were sent to LC Sciences (Hangzhou, China) for RNA-seq library preparation. After cluster generation, Transcriptome sequencing was carried out on an Illumina Novaseq ${ }^{\mathrm{TM}} 6000$ platform that generated raw reads. The data that support the findings of this study have been deposited in the CNSA (https://db.cngb.org/cnsa/) of CNGBdb with accession number CNP0000970. After removing adaptor sequences, ambiguous ' $\mathrm{N}$ ' nucleotides (with the ratio of ' $\mathrm{N}$ ' greater than $5 \%$ ) and low-quality sequences (with a quality score less than 10), the remaining clean reads were assembled using Trinity software as described for de novo transcriptome assembly with a reference genome. The mapped clean-read number was normalized to RPKM (reads per kilo of per million mapped reads). We used the edgeR package to determine the StringTie genes. Threshold of significant difference was $\mid \log 2$ foldchange $\mid \geq 1, p<$ 0.05 . The Gene Ontology (GO) enrichment analysis, Kyoto Encyclopedia of Genes and Genomes (KEGG) pathway, Heatmap analysis, and VolcanoPlot analysis were conducted at https://www.lc-bio.cn/ (LC Sciences, Hangzhou, China).

\section{II-1 $\beta$ analysis}

Commercial enzyme-linked immunosorbent assay (ELISA) kits (Beyotime, Shanghai, China) were used for measuring II- $1 \beta$ levels in cortical tissue or supernatant as per the manufacturer's instructions.

\section{Immunofluorescence analysis}

Paraffin section of brain tissue was obtained after transcardial perfusion and primary cortical microglia was fixed by $4 \%$ paraformaldehyde after LPS + ATP treatment, then followed by overnight incubation with primary antibodies: primary anti-Cd11b antibodies (ab184308, Abcam, Cambridge, UK), anti-Gfap (\#80788, CST, MA, USA), anti-Nlrp3 antibodies (19771-1-AP, Proteintech, Wuhan, China), anti-caspase1 antibodies (22915-1-AP, Proteintech), anti-caspase6 antibodies (ab185645, Abcam), anti-II-1 $\beta$ (\#12242, CST), and anti-a-tubulin (\#3873, CST), and then incubated with secondary antibodies. Cell nuclei were stained with DAPI, 6 random high-power fields were chosen and images were obtained using a fluorescence microscope (Leica, Oskar-Barnack, Germany).

\section{Co-immunoprecipitation (CoIP)}

Samples of cortical tissue or primary cortical microglia were lysed using a Pierce IP lysis buffer (Thermo Scientific) with a proteinase inhibitor cocktail (Roche, Basel, Switzerland). After centrifugation, suspensions were collected. ColP was performed using a Dynabeads ${ }^{\mathrm{TM}}$ Co-Immunoprecipitation Kit (Thermo Scientific), anti-Nlrp3 (ab263899, Abcam), and anti-Asc (\#67824, CST) antibodies were coupled to Dynabeads, and IgG served as the negative control. The total proteins were mixed with antibody-coupled Dynabeads and incubated overnight at $4{ }^{\circ} \mathrm{C}$. Adsorbed Dynabeads were washed with wash buffer, and bound proteins were eluted with $20 \mu \mathrm{L}$ eluent, mixed with $20 \mu \mathrm{L} 2 \times$ Laemmli buffer, and then boiled for $5 \mathrm{~min}$. Finally, samples were loaded onto $4 \%-20 \%$ BeyoGel $^{\text {TM }}$ Plus PAGE (Beyotime) for electrophoresis.

\section{Western blot analysis}

The total protein from cortical tissue or primary cortical microglia was lysed by the lysis buffer (Sigma-Aldrich), and a BCA protein kit (Thermo Scientific) was used to quantify protein concentration. Following SDS-PAGE electrophoresis and western transfer, a PVDF membrane (Millipore, Billerica, MA, USA) was blocked with 5\% bovine serum albumin (Sigma-Aldrich) and incubated at $4{ }^{\circ} \mathrm{C}$ for overnight with the primary antibodies: Pgam5 (ab131552, Abcam), Nlrp3 (ab263899, Abcam), caspase1 (ab179515, Abcam), caspase1 (p20) (AG-20B-0042-C100, AdipoGen), Asc, Gsdmd (Gasdermin-D) (ab219800, Abcam), caspase8 (\#4790, CST), Cleaved caspase8 (\#8592, CST), II-1 $\beta$ (\#12242, CST), Cleaved II-1 $\beta$ (\#63124, CST), and Gapdh (\#2118, CST).

For chemical cross-linking, the monomer and oligomer of Asc (\#67824, CST) and Pgam5 (ab131552, Abcam) were detected as described by Fernandes-Alnemri and his colleagues [22]. Then crude pellet was resuspended in CHAPS buffer and was chemically crosslinked with $4 \mathrm{mM}$ non-cleavable disuccinimidyl suberate (DSS) cross-linker for $30 \mathrm{~min}$. The samples obtained were used for Western blot analysis.

\section{qRT-PCR analysis}

The total RNA was isolated using Trizol reagent (Invitrogen, Waltham, MA, USA). A HiFi-MMLV cDNA First-Strand Synthesis Kit (CW Bio, Beijing, China) was used for reverse transcription. GoTaq qPCR Master Mix (Promega, WI, 
USA) was used for qRT-PCR analysis on the CFX96TM real-time system (BioRad, CA, USA). The expression of the genes of interest was normalized to the levels of Gapdh. Primer sequences are listed in Table S1:

\section{Primary cortical microglia culture}

Pure neonatal microglia cultures were obtained from the cortices of neonatal Pgam5 $5^{-1-}$ or WT mice as previously described in detail [23]. Briefly, cortices were incubated in trypsin/EDTA solution for $15 \mathrm{~min}$ at $37{ }^{\circ} \mathrm{C}$, and then the culture medium (DMEM with the addition of $10 \%$ fetal calf serum, $1 \%$ penicillin/streptomycin, and $2 \mathrm{mM} \mathrm{L-glutamine)} \mathrm{was} \mathrm{added}$ to stop the reaction. After dissociated the cortices, the suspension was centrifuged at $1000 \mathrm{rpm}$ for $2 \mathrm{~min}$. Cells were resuspended in culture medium and grown in $75 \mathrm{ml}$ flasks at $37{ }^{\circ} \mathrm{C}$ with $5 \% \mathrm{CO}_{2}$, while changed the culture medium every 3 days. After 2 weeks in culture, the cells were isolated by gently shaking of the flask $(250 \mathrm{rpm})$ at $37^{\circ} \mathrm{C}$ for $1 \mathrm{~h}$ to detach microglia, and collected the medium and immediately centrifuged for $2 \mathrm{~min}$ at $1200 \mathrm{rpm}$, and the obtained pure microglia pellet was resuspended in fresh culture medium and seeded into subcultures. About $95 \%$ of these cells were positive for C $111 b$, a marker for microglia cell types. Experiments were started $24 \mathrm{~h}$ after cultivation.

\section{Transfection in primary cells}

Pgam5-shRNA Lentiviral Particles (sc-152184-V; Santa, CA, USA), Rip3shRNA Lentiviral Particle (sc-61483-V; Santa), and caspase8-shRNA Lentiviral Particles (sc-37226-V; Santa) were used for transfection following the manufacturer's instructions in primary cortical neurons or microglia. At $48 \mathrm{~h}$ after infection, transfection efficiency was assessed using qRT-PCR analysis (Fig. S2).

\section{Microglial viability and activation}

$10 \mathrm{ng} / \mathrm{mL}$ LPS (Sigma-Aldrich) $+5 \mathrm{mM}$ ATP (Sigma-Aldrich) were used to in vitro [16]. After LPS treatment for $4 \mathrm{~h}$ and ATP for an additional 10, 20, 30 , or $45 \mathrm{~min}$, cells and medium supernatant were collected for subsequent detections. Cell Counting Kit-8 (CCK8; Dojindo Laboratories, Tokyo, Japan) was used to determine cell viability. Griess reaction (Solarbio, Beijing, China) to measure the NO production in microglia.

\section{Asc speck staining}

Cells were blocked with $5 \%$ goat serum after fixation and permeabilization, then incubated with anti-Asc antibody $\left(\# 67824\right.$, CST) at $4{ }^{\circ} \mathrm{C}$ overnight and with secondary antibody (Alexa Fluor 594-conjugated, Abcam) at $37^{\circ} \mathrm{C}$ for $2 \mathrm{~h}$. After stained cytoblast with DAPI, images were collected using an inverted fluorescence microscope (Olympus).

\section{Statistical analysis}

All the data were presented as the mean \pm SEM and were analyzed using SPSS statistical software (version 22.0, IBM, Armonk, NY, USA) and GraphPad Prism 5 software (San Diego, CA, USA). Sample size and animal numbers were determined based on previous studies. Before the analysis, all data were checked for normality and homogeneity of variances. Twotailed independent-sample $t$-test and one-way and two-way analysis of variance (ANOVA) followed by Tukey's post hoc multiple comparison tests were used. The variance is similar between the groups that are statistically compared. A $p$ value $<0.05$ was considered to be statistically significant.

\section{RESULTS}

\section{Pgam5 depletion relieves neurological deficits and nerve injury after TBI}

In Pgam5 ${ }^{-1-}$ mice, the mRNA and protein levels of Pgam5 were near negligible in comparison to WT mice (Fig. 1A). Before the tests, no differences in mNSS, Rotarod, or open field scores were observed between the groups. 7 days after TBI, Pgam $5^{-1-}$ mice showed lower mNSS scores compared to WT mice (Fig. 1B). In the Rotarod test (Fig. 1C), WT-TBI mice were easy to fall off the stick, but the time of latency to fall of Pgam5 ${ }^{-1-}-\mathrm{TBI}$ mice was better compared to the WT-TBI mice. In the open field test (Fig. 1D), WT-TBI mice significantly moved less in the perimeter region resulting in less total distance, and Pgam5 $5^{-1-}$-TBI mice showed a significant increase in the activity of the perimeter region and the total distance.
TBI induced significant brain tissue edema, the score was maximal at $2 \mathrm{~d}$ and gradually decreased, but still with significant difference compared to the sham groups (Fig. 1E). Pgam5 deficiency significantly alleviated this phenomenon compared with WT-TBI mice from day 2 (Fig. 1E). T2-weighted MRI images were also used to evaluate the effect of Pgam5 deletion at $48 \mathrm{~h}$ post-TBI (Fig. 1F) and Pgam5 deficiency alleviated histological impairments. The TUNEL/NeuN positive cells were significantly enhanced in WT-TBI mice, but that significantly reduced in Pgam5 ${ }^{-1-}$-TBI mice (Fig. 1G). After TBI, the damaged neuron cells had increased, presenting extensive degenerative changes, including less Nissl body staining. Inversely, severe nerve injury was seen to be significantly reduced in Pgam5 $5^{-1-}-\mathrm{TBI}$ mice (Fig. 1H). Pgam5 depleted blocked the reduction in the arborization of neurites after TBI (Fig. 11).

\section{Pgam5 facilitates II-1 $\beta$ activation and microglia activation after TBI}

To classify the biological function of the differentially expressed genes (DEGs), a GO enrichment analysis was carried out. As shown in Fig. 2A, the enriched DEGs were mainly associated with the inflammatory response (GO:0006954), and the extracellular region (GO:0005576). The KEGG enrichment analysis showed that the cytokine-cytokine receptor interaction (ko04060), NF-kappa B signaling pathway (ko04064), Tnf signaling pathway (ko04668), and II-17 signaling pathway (ko04657) were enriched (Fig. 2B), indicating that Pgam5 in regulating inflammatory response through regulating these genes' expression patterns. To assess the role of Pgam5 on neuroinflammation, 1,076 genes associated with inflammatory response were isolated and analyzed. 3 genes were upregulated and 37 genes were down-regulated when WT-CtI were compared to WT-TBI; 6 genes were up-regulated and 80 genes were down-regulated when WT-TBI were compared to Pgam5 ${ }^{-1-}$-TBI (Fig. 2C). Heatmap analysis showed TBI decreased Dsc2, Traf1, and Fsd1I mRNA levels but induced II-1 $\beta$, Hmox1, Cxcl10, and NIrp3. expression (Fig. 2D). Interestingly, Pgam5 deletions did not mediate II-1 $\beta$, Hmox1, Cxcl10, and Nlrp3. mRNA levels (Fig. 2D), that might regulate inflammatory responses by affecting the post-translational modification and protein expression of related genes, including Nlrp3, caspase1, Gsdmd, and II-1 $\beta$.

As shown in Fig. 3A, pyroptosis and inflammation molecules were still high mRNA levels Pgam5 $5^{-1-}$-TBI mice after TBI. II-1 $\beta$ mRNA level in cortex significantly increased within $48 \mathrm{~h}$ after TBI, mRNA levels peaked at $12 \mathrm{~h}$, and then it went down (Fig. 3A). However, the mRNA level was still $23.7 \pm 4.6$-fold relative change at $12 \mathrm{~h}$ in Pgam5 ${ }^{-1-}-\mathrm{TBI}$ mice (Fig. 3A). Inversely, II-1 $\beta$ levels peaked $2427 \pm 434 \mathrm{pg} / \mathrm{mg}$ at $48 \mathrm{~h}$ and then gradually declined after TBI, while this was significant in the Pgam $5^{-1-}$-TBI group (Fig. 3B). However, Pgam5 deficiency did not affect the pyorptosisrelated gene expression levels (Fig. 3C), which was consistent with the result of RNA-seq. Pgam5 level was at the same tendency with II-1 $\beta$ level and appeared to peak at 2 days after TBI (Fig. 3D). The data suggested that II-1 $\beta$ might be linked to Pgam5. TBI triggered the recruitment of Pgam5 to Nlrp3 complex, and Pgam5 deficiency suppressed the assembly of Nlrp3 inflammasome, including NIrp3 interacting with caspase 1 and Asc, and II-1 $\beta$ was regulated by Nlrp3-mediated pyroptosis (Fig. 3E). Furthermore, a high level of $\mathrm{Cd} 11 \mathrm{~b}$ appeared around the damaged cortical tissue after $\mathrm{TBI}$, and $\mathrm{TBI}$ increased the interaction with NIrp3 and caspase1 (Fig. 3F), and induced $\mathrm{Cd} 11 \mathrm{~b}$ and Gfap positive cells, indicating the activation of microglia and astrocytes (Fig. 3G). In Pgam5 ${ }^{-l-}$-TBI mice, $\mathrm{Cd} 11 \mathrm{~b} / \mathrm{Il}-1 \beta$ positive cells were significantly less and the interaction with Nlrp3 and caspase1 was blocked compared with the WT-TBI group (Fig. 3F-H). The data suggest that Pgam5 facilitates microglia activation-mediated $I I-1 \beta$ and 
A

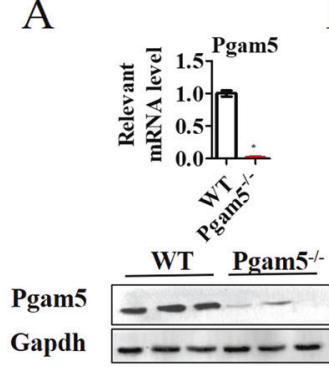

B
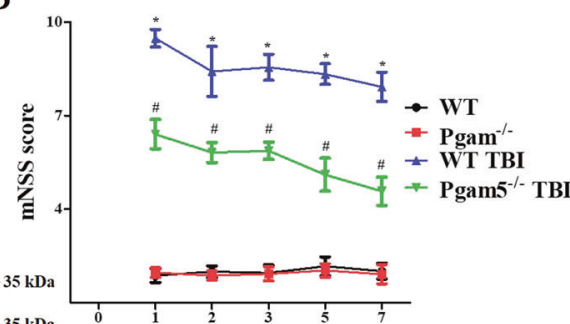

$\mathrm{C}$

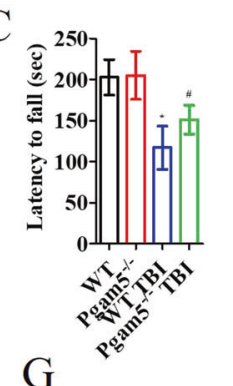

$\mathrm{D}$
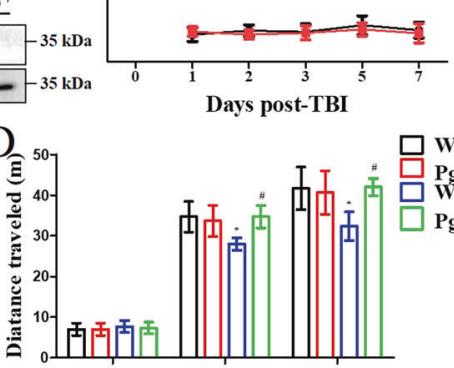

G

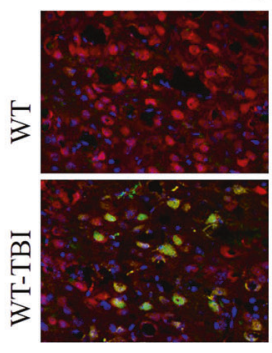

WT

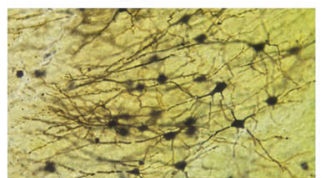

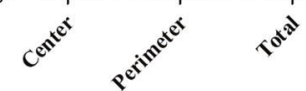

DAPI/NeUN/TUNEL

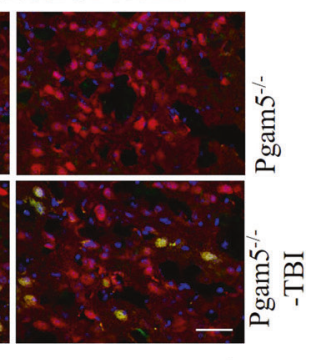

Pgam5 $^{-/-}$

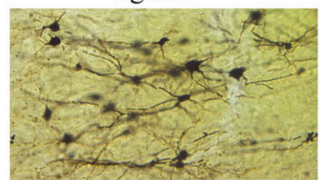

$\mathrm{E}$

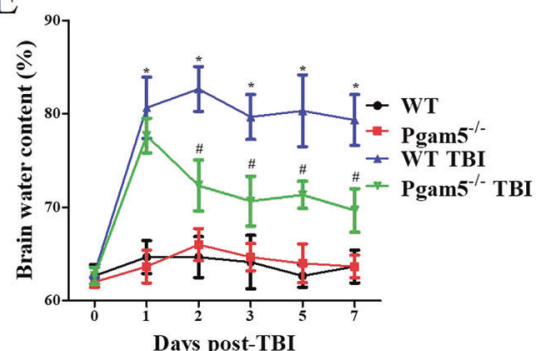

Days post-TBI

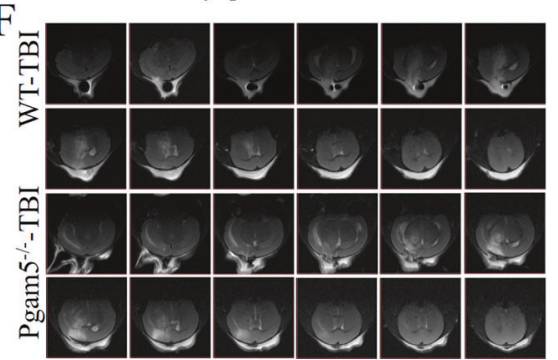

$\mathrm{H}$

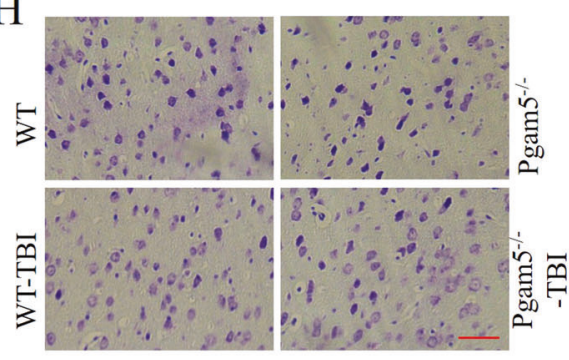

WT-TBI

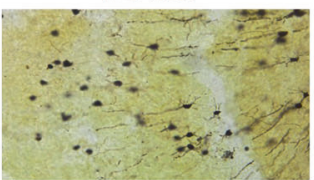

Pgam5 $^{-/-}$-TBI

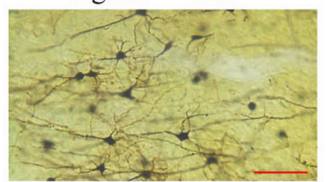

Fig. 1 Pgam5 deficiency improves neurological deficits, neuron damage, and neuronal neurites degeneration after TBI. A Western blot and RT-PCR results showed that Pgam5 expression was abolished in Pgam5 ${ }^{-1-}$ mice. Neurological performances were assessed by (B) mNSS, (C) Rotarod test, and (D) open field test, $n=8$. E At 1, 2, 3, 5, and 7 days after TBI in WT and Pgam5 ${ }^{-1}$ mice, the brain water content was detected. F At 2 days after TBI, the T2-weighted MRI images showed histological impairments. G Cortical injuries to coronal sections were assessed using TUNEL staining $48 \mathrm{~h}$ post-TBI. Nuclei were stained with DAPI and neurons were stained with NeuN. $\mathbf{H}$ Nissl staining was performed to determine the morphological changes and damage of cortex neurons after TBI. I Representative pictures from cortical sections show prominent Golgi-Cox staining of degenerating neurites 2 days post-TBI. The images shown are representative of typical images from 6 mice in each group. E I, $n=6$. Data shown are means \pm SEM, ${ }^{*} p<0.05$ compared to the WT group and ${ }^{\#} p<0.05$ compared to WT TBI group by two-way ANOVA followed by Tukey's post hoc multiple comparison test.

acute inflammation, which may be involved in the pathology of nerve damage.

\section{Pgam5 is required for microglia-induced II-1 $\beta$ secretion via activating Asc oligomerization}

There was a significant decrease in nitrite in LPS-induced Pgam5 $5^{-1-}$, Rip3-shRNA, and caspase8-shRNA microglia compared with WT microglia (Fig. 4A). LPS + ATP suppressed cell viability in WT microglia but not in Pgam5 ${ }^{-1-}$, Rip3-shRNA, and caspase8-shRNA microglia (Fig. 4B). As shown in Fig. 4C, LPS + ATP significantly induced $\|-1 \beta$ secretion, while $\|-1 \beta$ secretion was significantly suppressed in Pgam5 $^{-1-}$ microglia after stimulation related to LPS + ATP-induced WT microglia but not in Rip3-shRNA and caspase8-shRNA microglia (Fig. 4C). Time-course analysis showed that LPS-induced II- $1 \beta$ secretion by Pgam $5^{-/-}$was reduced at all time points tested, but not influenced by Rip3-shRNA and caspase8shRNA (Fig. 4D). LPS + ATP stimulation activated the Nlrp3 inflammasome in primary microglia, which manifested as caspase1 p20 and II-1 $\beta$ p17 secretion in an Nlrp3/Asc-dependent manner. Reduced levels of activated caspase1 (p20) and II-1 $1 \beta$ p17 were measured in LPS + ATP-induced Pgam5 ${ }^{-1-}$ microglia (Fig. 4E). Indeed, caspase 8 was activated in Pgam5 $5^{-1-}$ and WT microglia, and Rip3 or caspase8 downregulation does little to regulate caspase1 p20 and IL-1 $\beta$ p17 secretion in LPS + ATP-induced microglia (Fig. 4E). Pgam5 downregulation reduced mature II- $1 \beta$ secretion, but not mRNA levels of II-1 $\beta$ and other inflammatory cytokines (Fig. 4F). LPS-induced high levels of pyroptosis-related genes were still in Pgam5 $5^{-1-}$ microglia (Fig. 4G).

ColP results showed that interaction with Asc and Nlrp3, caspase1, and caspase8 were significantly decreased in Pgam5 $5^{-/-}$ microglia compared with WT microglia, but caspase8-shRNA did not affect this phenomenon (Fig. 4H). In WT microglia, LPS + ATPinduced Asc oligomerization was measured concomitant with the appearance of caspase1 p20 (Fig. 4l). ASC translocation to an insoluble compartment and Asc oligomerization were significantly reduced in Pgam5 ${ }^{-1-}$ microglia, but weak variation was seen in 
A

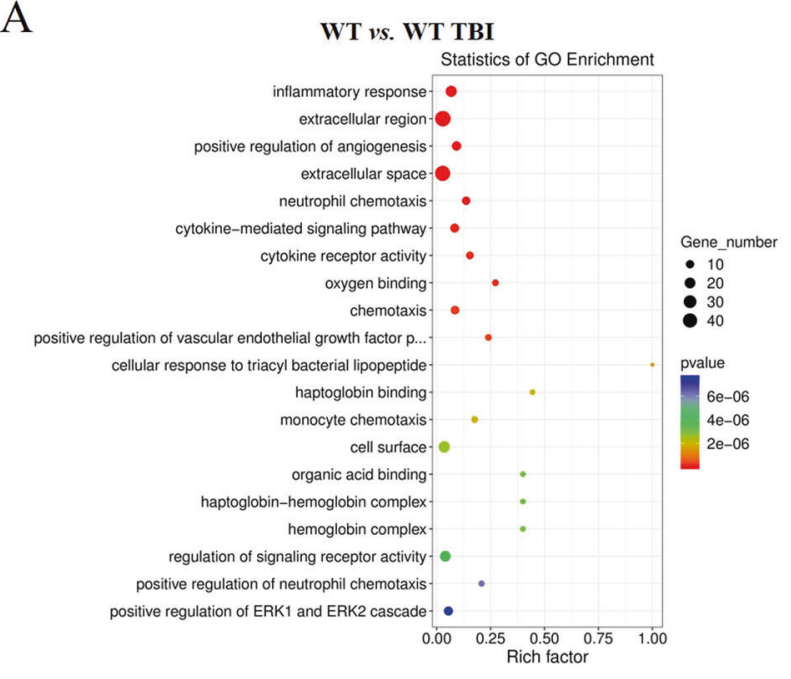

$\mathrm{B}$

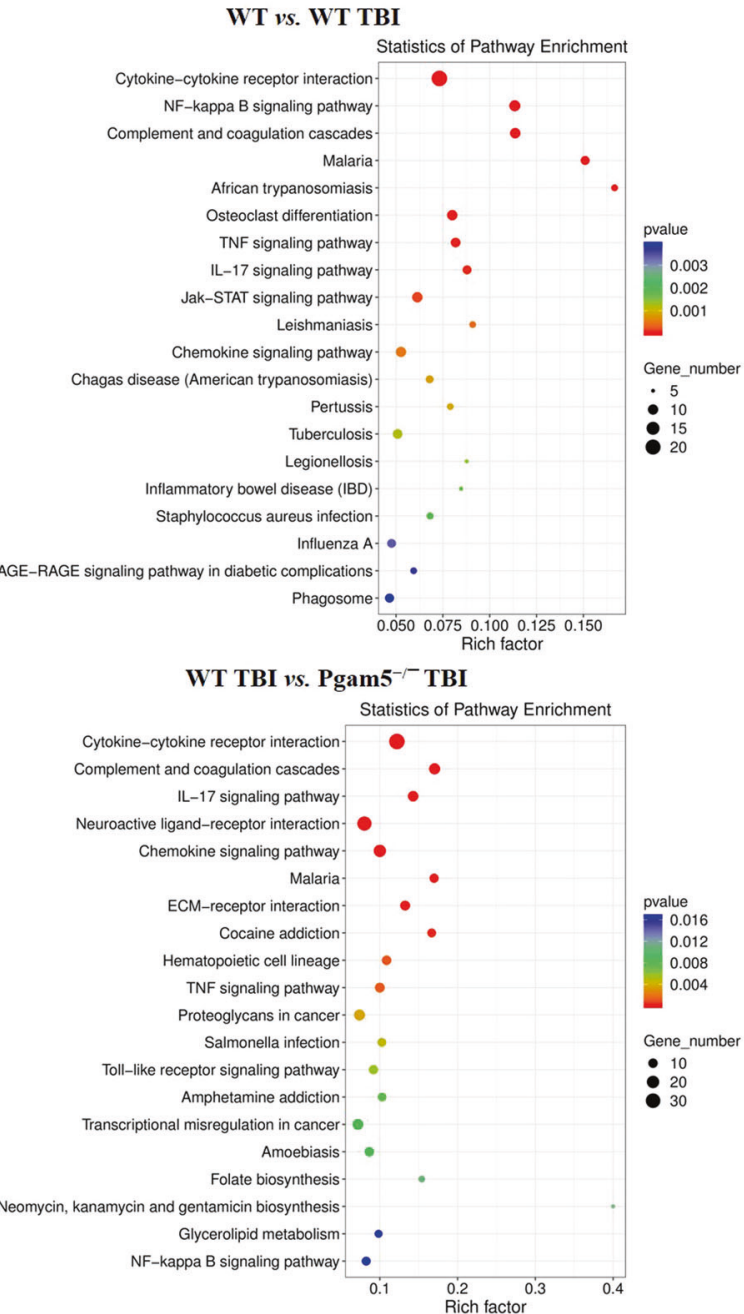

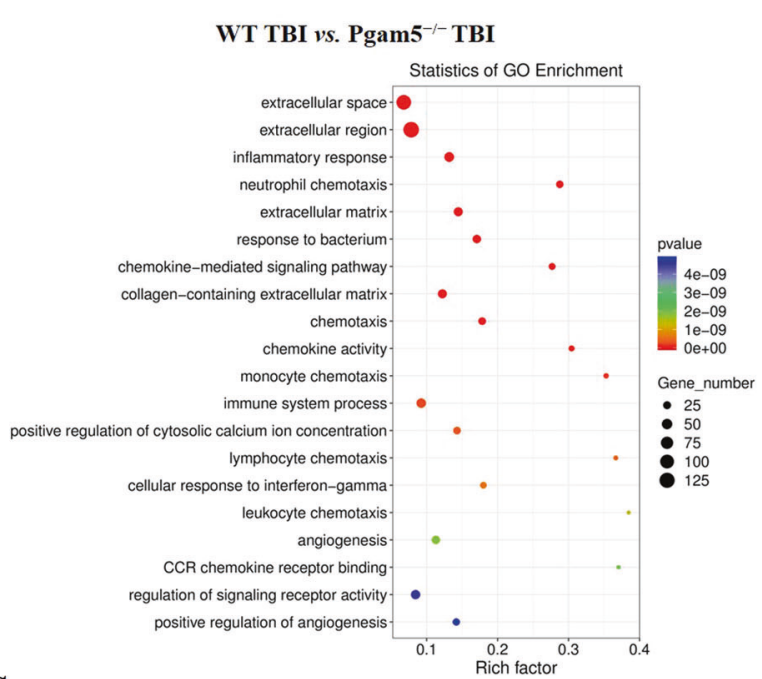

$\mathrm{C}$

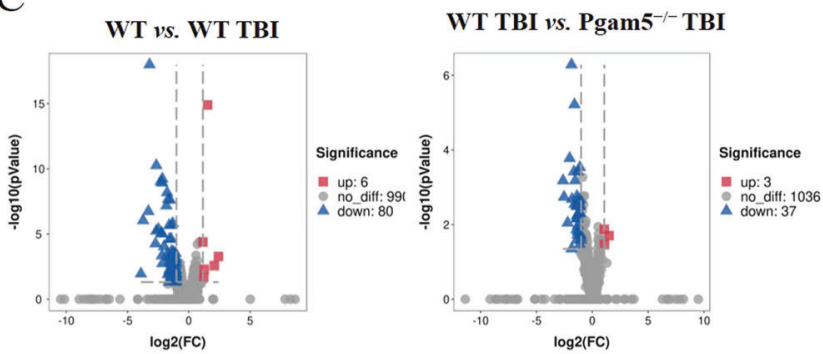

$\mathrm{D}$

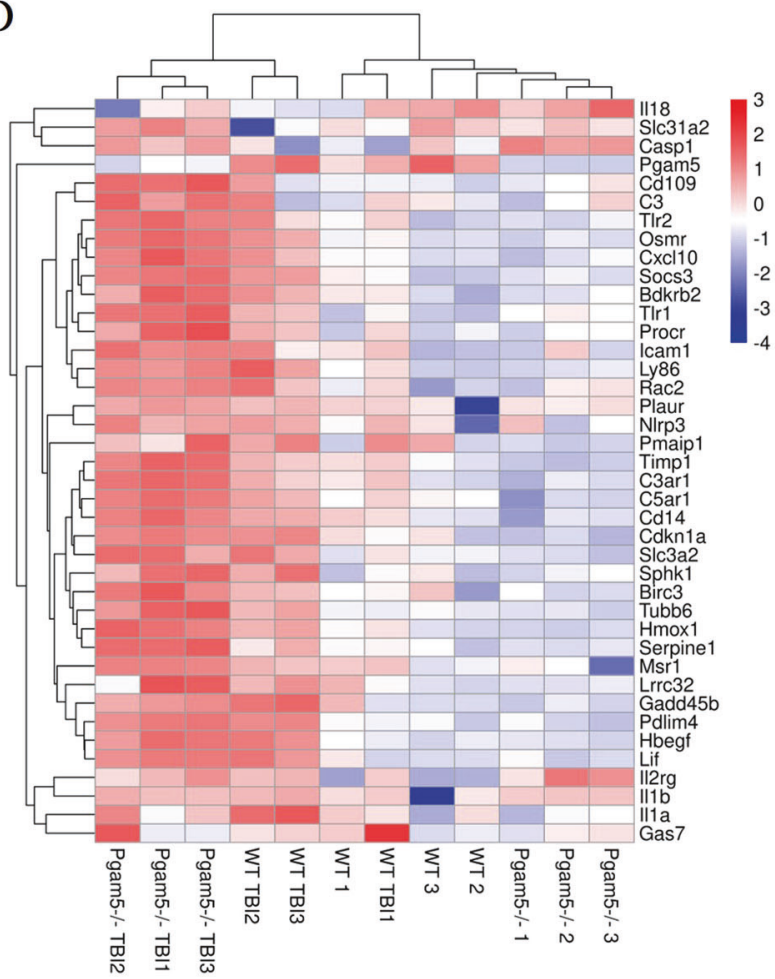

Fig. 2 RNA-seq analysis of cortical tissue. Total 55,450 genes of cortical tissues were detected by LC Sciences for RNA-seq analysis. A GO enrichment analysis was carried out to classify the biological function of DEGs. Each box shows the GO term number, the p-value, and GO term. B KEGG pathway enrichment analysis for DEGs. The top 20 KEGG pathways are shown. The box color indicates the level of statistical significance. The dot size means the gene number. C A 1076 genes associated with inflammatory response were isolated and analyzed by VolcanoPlot. D Heatmap analysis of inflammation-related DEGs between WT group, WT TBI group, Pgam5 $5^{-1-}$ group, and Pgam5 ${ }^{-1-}$ TBI group. Only the top 40 genes were included in the DEGs heatmap. 
A

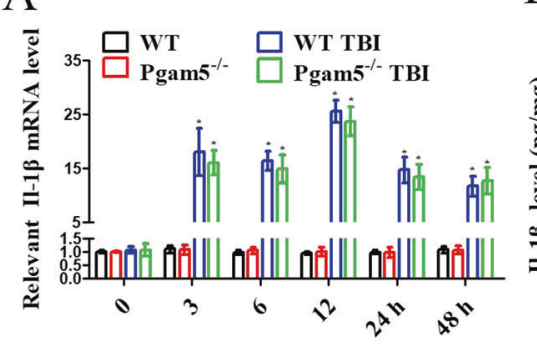

C

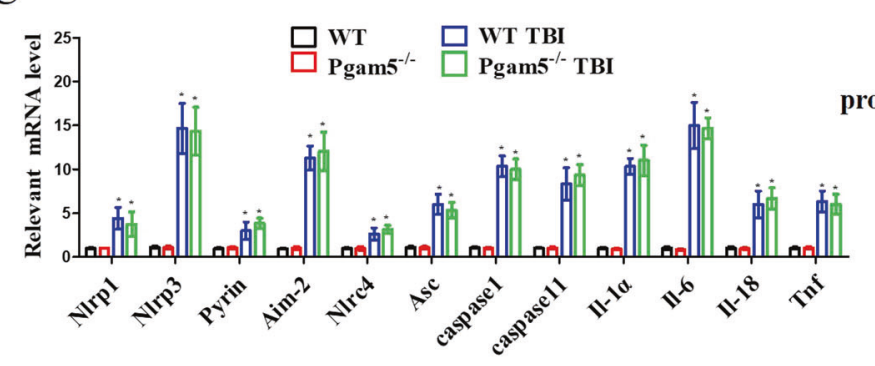

B

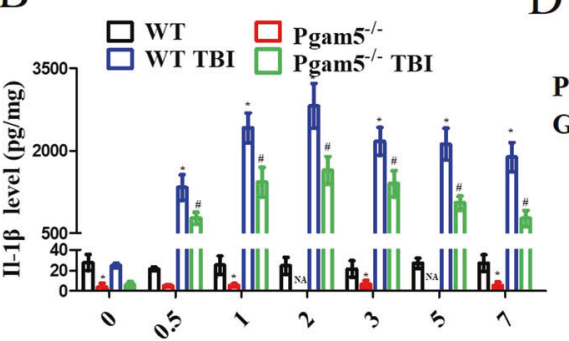

D

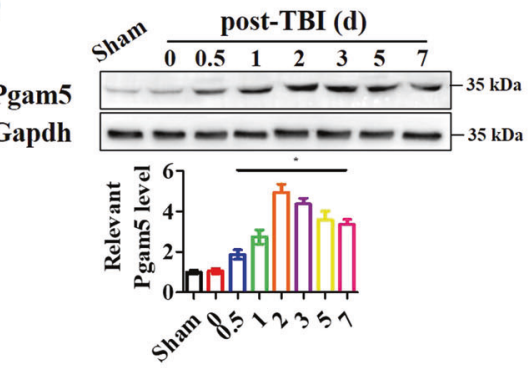

F DAPI/NIrp3/caspase1
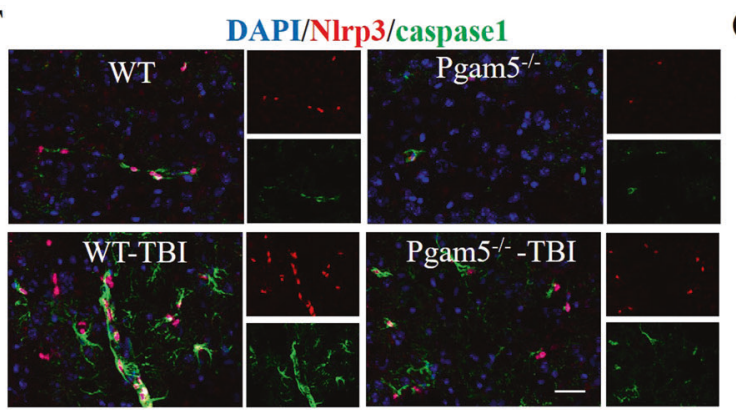

G

E Pgam5 $\frac{+/+}{-+} \frac{-/-}{-+}$

Pgam5 $+/+$

NIrp3

pro-caspase1 $-200 \mathrm{kDa}$

p20 $\rightarrow \ldots+\ldots$

Asc

Gsdmd pro-Il-1ק

p17

Gapdh
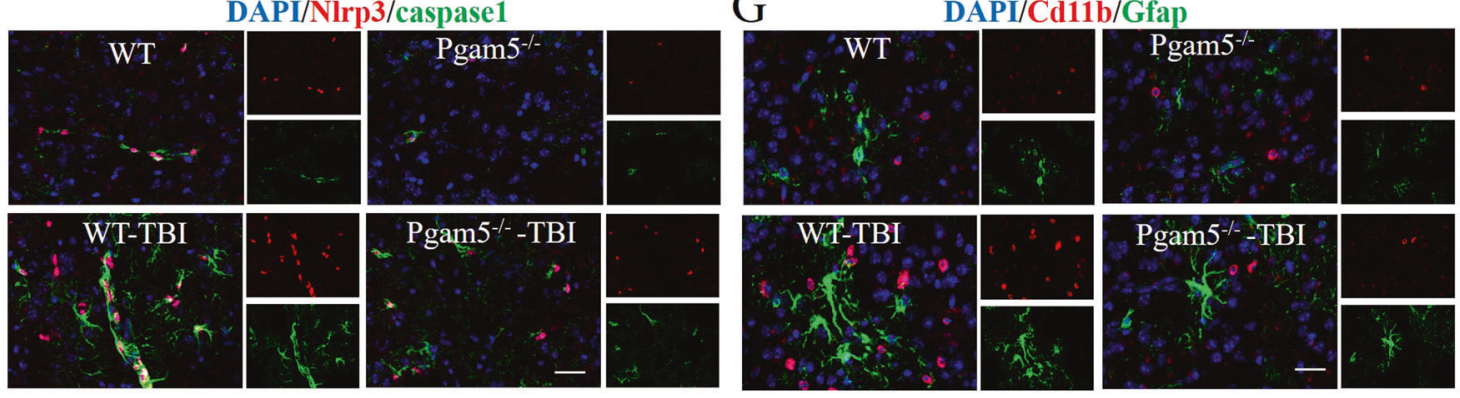

$\mathrm{H}$
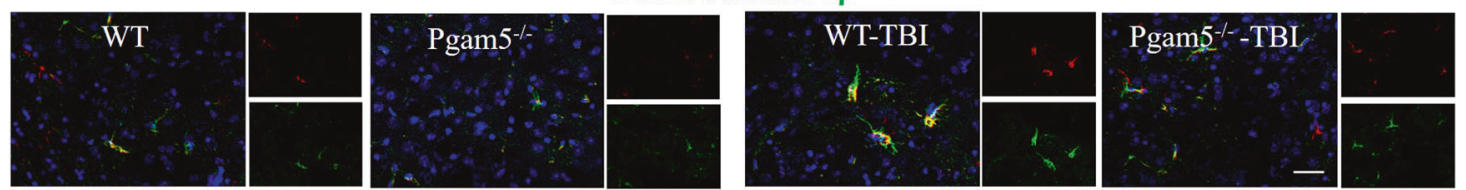

Fig. 3 Pgam5 depletion relieves TBI-induced II-1 $\beta$ through NIrp3 inflammasome activation and Pgam5 is involved in TBI-induced microglial activation in vivo. The II- $1 \beta$ level was monitored after TBI in WT and Pgam5 ${ }^{-1-}$ mice. A qRT-PCR analysis of II-1 $\beta$ mRNA level and (B) ELISA analysis of II- $1 \beta$ level. C Nlrp1, Nlrp3, Pyrin, Aim-2, Nlrc4, Asc, caspase1, caspase11, II-1 $\alpha,\|-6\|-$,18 , and Tnf mRNA in Pgam5 ${ }^{-1-}-$ TBI mice $^{2}$ were measured at $24 \mathrm{~h}$ after TBI. (A, B and C) ${ }^{*} p<0.001$ compared to the WT group and \#p<0.001 compared to the WT TBI group by two-way ANOVA followed by Tukey's post hoc multiple comparison test. D Western blot analysis of Pgam5 expression during 7 days after TBI in the cortex. The lysate of the cortex was analyzed using Western blot assay to detect the Nlrp3/caspase $1 / \mathrm{ll}-1 \beta$ pathway at $48 \mathrm{~h}$ after TBI. ${ }^{*} p<0.001$ compared to the sham group by one-way ANOVA followed by Tukey's post hoc multiple comparison test. E Results of immunoblotting with antibodies to the indicated proteins and immunoprecipitated with anti-Nlrp3. At $48 \mathrm{~h}$ after TBI in WT and Pgam5 ${ }^{-1-}$ mice, cortical sections were analyzed by immunofluorescence for the expression of (F) Nlrp3/ caspase1, (G) Cd11b/Gfap, and (H) Cd11b/II-1ß. Nuclei were stained with DAPI. Scale bar: $20 \mu \mathrm{m}$. Data shown are means $\pm \mathrm{SEM}, n=3$.

caspase8-shRNA microglia (Fig. 4l). Furthermore, LPS + ATP drove Pgam5 translocation to the insoluble compartment and Pgam5 oligomerization (Fig. 4l). After stimulation of LPS or LPS + ATP in microglia, large intracellular Asc specks were rapidly formed in the cytosol. Asc specks were reduced in Pgam5 $5^{-/-}$microglia, but weak variation was seen in caspase8-shRNA microglia (Fig. 4J). Although some reports have shown that caspase8 participates in the processing of pro-II-1 $\beta$ under certain conditions [24, 25]. the data suggest that Pgam5 facilitates the Asc-dependent pro-II-1 $\beta$ processing via caspase1-associated inflammasome that is independent of Rip3/caspase8 pathway in LPS + ATP-stimulated microglia.

\section{DISCUSSION}

Microglia are the primary mediators of the innate immune response in CNS, and the proinflammatory cytokines released by microglia, such as $I I-1 \beta$ and Tnf-a, are important assessment indices of microglia polarization and pro-inflammatory after TBI [24]. Indeed, inhibiting microglia activation-mediated inflammation is suggested to improve the neurological outcomes post-TBI [25]. In this study, we have investigated the molecular mechanism of Pgam5 after TBI. Pgam5 deletion reduced TBI-induced NIrp3/ caspase $1 / / 1-1 \beta$ pathway activation, microglial activation, and neurological deficits in vivo. Pgam5 facilitated Asc polymerization 
A

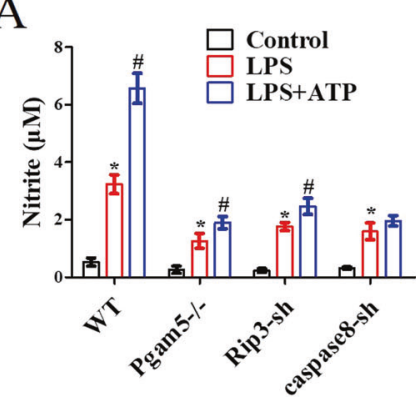

$\mathrm{D}$

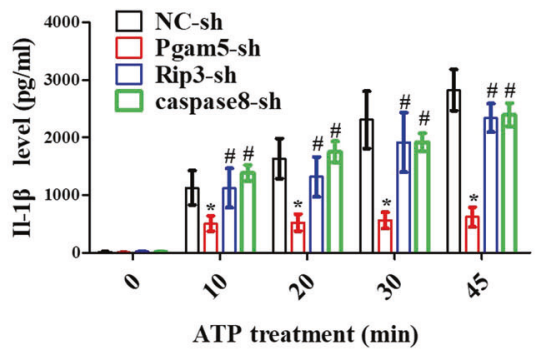

$\mathrm{B}$

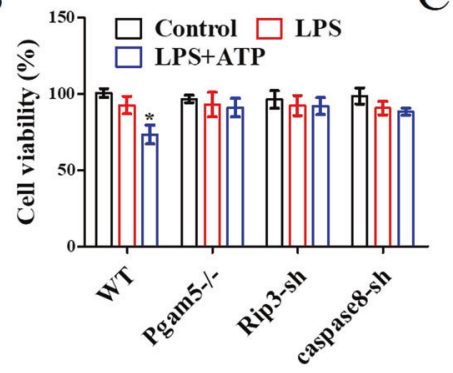

$\mathrm{C}$

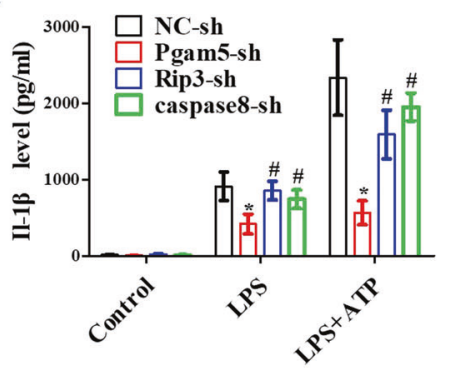

E

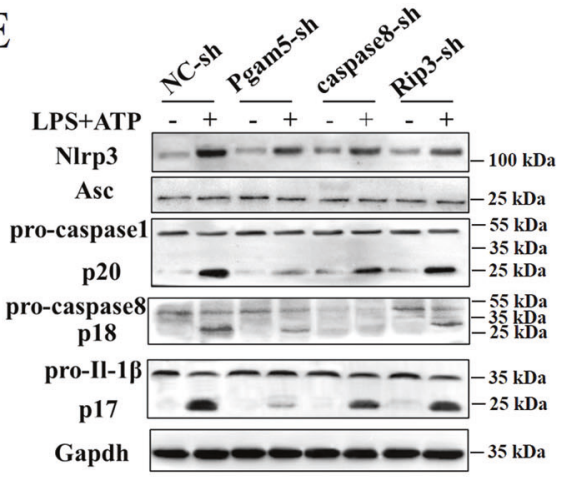

$\mathrm{F}$
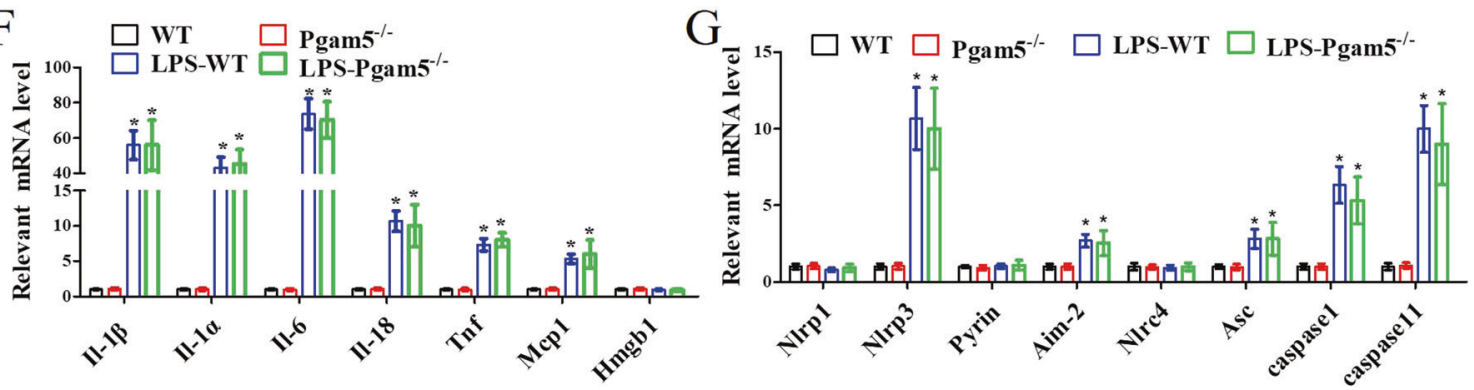

$\mathrm{H}$

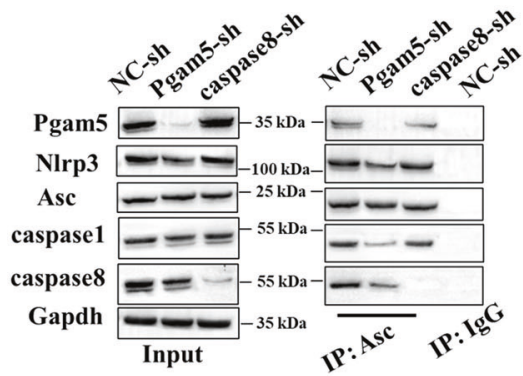

$\mathrm{J}$

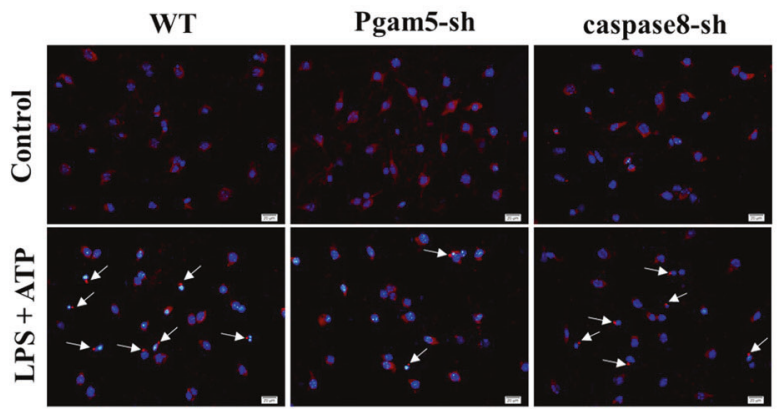

I
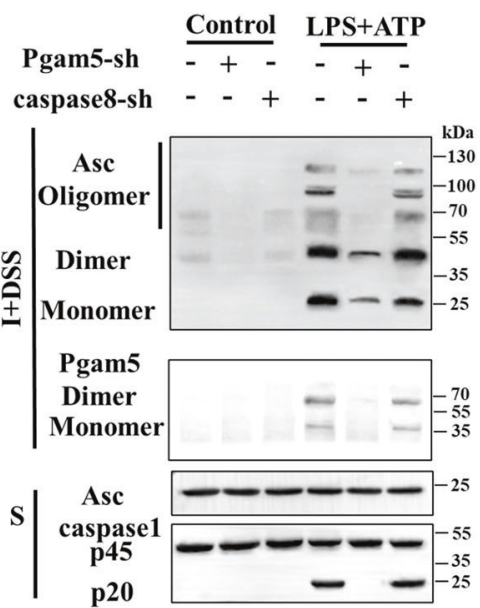

and $I 1-1 \beta$ secretion in microglia, and Pgam5 downregulation alleviated LPS + ATP-induced microglia activation and II-1 $\beta$ via Asc/caspase1-mediated pyroptosis.

Neuroinflammation is one of the leading causes of secondary brain injury, and its reduction is regarded as an excellent choice to improve the prognosis of TBI $[26,27]$. In clinical practice, Helmy et al. have demonstrated that the injection of recombinant IL-1R antibody reduces IL-1-mediated brain injury post-TBI by alleviating neuroinflammation [28]. Moriwaki et al. have verified that Pgam5 promotes inflammasome activation including $I I-1 \beta$ secretion in 
Fig. 4 Pgam5 is required for microglia-induced II-1 $\beta$ secretion and Asc oligomerization. NO production was detected by the (A) Griess reaction and cell viability was measured by (B) CCK8 assay. (A) ${ }^{*} p<0.001$ compared to the control group and ${ }^{\#} p<0.001$ compared to the LPS group, by two-way ANOVA followed by Tukey's post hoc multiple comparison test. C Primary microglia were primed with LPS for $4 \mathrm{~h}$ prior to stimulation with ATP for $30 \mathrm{~min}$, and II- $1 \beta$ secretion was determined by ELISA. D Dynamically monitor II-1 $\beta$ level in LPS-primed microglia treated with ATP for the indicated amount of time. C, D ${ }^{*} p<0.001$ compared to the NC-sh group and ${ }^{\#} p<0.001$ compared to the Pgam5 ${ }^{*}$ sh group, by two-way ANOVA followed by Tukey's post hoc multiple comparison test. (E) Whole-cell extracts were subjected to Western blot analysis. Microglia were stimulated with LPS + ATP and the mRNA of (F) cytokineand (G) pyroptosis-related genes were determined by qRTPCR. $p<0.001$ compared to the WT group by one-way ANOVA followed by Tukey's post hoc multiple comparison test. Data shown are mean \pm SEM $(n=4)$. Pgam5-shRNA and caspase8-shRNA microglia were stimulated with LPS + ATP. H Results of immunoblotting with antibodies to the indicated proteins and immunoprecipitated with anti-Asc. I The detergent-insoluble fraction was subjected to chemical cross-linking, and monomers, dimers, and oligomers of Asc and Pgam5 were measured. J Immunofluorescence analysis of Asc specks (arrows) in microglia. After treatments, cells were fixed and stained for Asc (Red). Nuclear staining with DAPI (Blue). $n=4$.

BMDMs [17]. Our results showed Pgam5 deficiency reduced NIrp3 inflammasome assembly, caspase 1 activation, and II- $1 \beta$ activation by inhibiting Asc polymerization in microglia, suggesting that Pgam5 also facilitated pyroptosis via Asc/caspase1 pathway.

As opposed to caspase8-mediated II-1 $\beta$ secretion and inflammation, Pgam5 has also been seen to be involved in LPS-induced II-1 $\beta$ secretion in caspase8-deficient BMDCs [29]. Moreover, caspase8 deficiency enhances LPS-induced NIrp3 inflammasome assembly and function, and Pgam5 contributes to Rip3-mediated induction of necrosis and Nlrp3 inflammasome activation in dendritic cells [29]. However, Moriwaki et al. have shown that primary mouse embryonic fibroblasts with Pgam5 deficiency responds normally to multiple inducers of apoptosis and necroptosis, and vesicular stomatosis virus-induced II- $1 \beta$ secretion is reduced in Pgam5 ${ }^{-/-}$BMDMs but not in BMDMs with Rip3 deficiency, suggesting that Pgam5-induced inflammation activation is independent of Rip3 [17]. Caspase1 inhibition prevents glial inflammasome activation and pyroptosis in cortical astrocytes and multiple sclerosis models $[30,31]$. VX-765 $(50 \mu \mathrm{M})$ suppresses caspase 1 activation and II-1 $\beta$ in astrocytes treated with unconjugated bilirubin but not pro-caspase1 level [30]. Our study demonstrated Pgam5 does not regulate the gene transcription of cytokines but rather participated in the processing of pro-II-1 $\beta$, and Pgam5 participated in Asc/caspase1-mediated microglia activation and pyroptosis. Phillips et al. have identified complex crosstalk and redundancy between caspase 1 and caspase 8 in II- $1 \beta$ processing in bone disease [32]. Kang et al. have demonstrated that caspase8 enzymatic activity is not required for dsRNAinduced Asc polymerization [33]. The results suggest that Pgam5 may be an upstream regulatory molecule of microglia in the Asc/ caspase $1 / \mathrm{Il}-1 \beta$ pathway under certain circumstances, such as infected BMDMs and nerve injury, which is independent of caspase8/Rip3 signal but via Asc polymerization.

Generally, Pgam5 is identified as a moderator dephosphorylating and activating Drp1, Bcl-xL, Fundc1, etc., which involves mitochondria fission and mitophagy to maintain normal function and state of mitochondria $[9,34]$. In this present work, Pgam5 depletion alleviated LPS-induced microglia activation and II- $1 \beta$ via Asc/caspase1-mediated pyroptosis. Numerous studies have shown that II-1 $\beta$ influences the survival of a variety of cells $[35,36]$, including cortical neurons and hippocampal neurons [31,36]. Cai et al. have demonstrated that intracerebral injection of II- $1 \beta$ leads to acute white matter and neuronal injury in neonatal rats [37], and Fan et al. have shown that $I I-1 \beta$ inhibition improves the associated neurological dysfunctions in juvenile rats [38].

\section{CONCLUSION}

In this study, we have discussed the mechanism of Pgam5 in nerve injury after TBI. Pgam5 facilitates microglial inflammasome activation to Asc/caspase1-generated II-1 $\beta$-mediated neuroinflammation after TBI, and TBI-induced neuroinflammation may be partly dependent on the interaction between Pgam5 and Nlrp3 inflammasome and Asc oligomerization. Thus, Pgam5 may be a potential therapeutic target to improve neuroinflammation and nerve injury after TBI.

\section{DATA AVAILABILITY}

The datasets and computer code produced in this study are available in the following databases, RNA-Seq data: Gene Expression, Omnibus CNP0000970 (http://db.cngb. org/cnsa/).

\section{REFERENCES}

1. Khellaf A, Khan DZ, Helmy A. Recent advances in traumatic brain injury. J. Neurol. 2019;266:2878-89.

2. Simon DW, McGeachy MJ, Bayir H, Clark RSB, Loane DJ, Kochanek PM. The farreaching scope of neuroinflammation after traumatic brain injury. Nat Rev Neurol. 2017;13:572.

3. Maas AIR, Menon DK, Adelson PD, Andelic N, Bell MJ, Belli A, et al. Traumatic brain injury: integrated approaches to improve prevention, clinical care, and research. Lancet Neurol. 2017;16:987-1048.

4. Shin N, Kim HG, Shin HJ, Kim S, Kwon HH, Baek H, et al. Uncoupled endothelial nitric oxide synthase enhances $\mathrm{p}$-Tau in chronic traumatic encephalopathy mouse model. Antioxid. Redox Signal. 2019;30:1601-20.

5. Wang ZM, Liu C, Wang YY, Deng YS, He XC, Du HZ, et al. SerpinA3N deficiency deteriorates impairments of learning and memory in mice following hippocampal stab injury. Cell Death Disco. 2020;6:88.

6. Lazaridis C, Rusin CG, Robertson CS. Secondary brain injury: Predicting and preventing insults. Neuropharmacology 2019;145:145-52.

7. Ma K, Zhang Z, Chang R, Cheng H, Mu C, Zhao T, et al. Dynamic PGAM5 multimers dephosphorylate $B C L-x L$ or FUNDC1 to regulate mitochondrial and cellular fate. Cell Death Differ. 2020;27:1036-51.

8. Yu B, Ma J, Li J, Wang D, Wang Z, Wang S. Mitochondrial phosphatase PGAM5 modulates cellular senescence by regulating mitochondrial dynamics. Nat Commun. 2020;11:2549.

9. Chen $G$, Han Z, Feng D, Chen $Y$, Chen $L, W u ~ H$, et al. A regulatory signaling loop comprising the PGAM5 phosphatase and CK2 controls receptor-mediated mitophagy. Mol Cell. 2014;54:362-77.

10. He GW, Günther C, Kremer AE, Thonn V, Amann K, Poremba C, et al. PGAM5mediated programmed necrosis of hepatocytes drives acute liver injury. Gut. 2017;66:716-23.

11. Lu W, Sun J, Yoon JS, Zhang Y, Zheng L, Murphy E, et al. Mitochondrial protein PGAM5 regulates mitophagic protection against cell necroptosis. PLoS One. 2016;11: e0147792.

12. Wang $Z$, Jiang $H$, Chen S, Du F, Wang $X$. The mitochondrial phosphatase PGAM5 functions at the convergence point of multiple necrotic death pathways. Cell. 2012;148:228-43.

13. Zhu H, Tan Y, Du W, Li Y, Toan S, Mui D, et al. Phosphoglycerate mutase 5 exacerbates cardiac ischemia-reperfusion injury through disrupting mitochondrial quality control. Redox Biol. 2021;38:101777.

14. Dong XH, Liu H, Zhang MZ, Zhao PX, Liu S, Hao Y, et al. Postconditioning with inhaled hydrogen attenuates skin ischemia/reperfusion injury through the RIPMLKL-PGAM5/Drp1 necrotic pathway. Am J Transl. Res. 2019;11:499-508.

15. Zhou H, Li D, Zhu P, Ma Q, Toan S, Wang J, et al. Inhibitory effect of melatonin on necroptosis via repressing the Ripk3-PGAM5-CypD-mPTP pathway attenuates cardiac microvascular ischemia-reperfusion injury. J. Pineal Res. 2018;65:e12503.

16. Chen $\mathrm{YH}$, Gong K, Xu QH, Meng J, Long TL, Chang CC, et al. Phosphoglycerate mutase 5 knockdown alleviates neuronal injury after traumatic brain injury through Drp1-mediated mitochondrial dysfunction. Antioxid. Redox Signal. 2021;34:154-70. 
17. Moriwaki K, Farias Luz N, Balaji S, De Rosa MJ, O'Donnell CL, Gough PJ, et al. The mitochondrial phosphatase PGAM5 is dispensable for necroptosis but promotes inflammasome activation in macrophages. J. Immunol. 2016;196:407-15.

18. O'Brien WT, Pham L, Symons GF, Monif M, Shultz SR, McDonald SJ. The NLRP3 inflammasome in traumatic brain injury: potential as a biomarker and therapeutic target. J. Neuroinflammation. 2020;17:104.

19. Liu W, Chen YH, Meng J, Wu MF, Bi FF, Chang CC, et al. Ablation of caspase-1 protects against TBI-induced pyroptosis in vitro and in vivo. J. Neuroinflammation. 2018;15:48.

20. Chen YH, Meng J, Bi FF, Li H, Chang CC, Ji C, et al. NEK7 regulates NLRP3 inflammasome activation and neuroinflammation post-TBI. Front. Mol. Neurosci. 2019;12:202.

21. Chen $\mathrm{YH}$, Meng J, Xu QH, Long TL, Bi FF, Chang CC, et al. Rapamycin improves the neuroprotection effect of inhibition of NLRP3 inflammasome activation after TBI. Brain Res. 2019;1710:163-72.

22. Fernandes-Alnemri T, Wu J, Yu JW, Datta P, Miller B, Jankowski W, et al. The pyroptosome: a supramolecular assembly of ASC dimers mediating inflammatory cell death via caspase-1 activation. Cell Death Differ. 2007;14:1590-604.

23. Vay SU, Flitsch LJ, Rabenstein M, Rogall R, Blaschke S, Kleinhaus J, et al. The plasticity of primary microglia and their multifaceted effects on endogenous neural stem cells in vitro and in vivo. J. Neuroinflammation. 2018;15:226.

24. Olde Heuvel F, Holl S, Chandrasekar A, Li Z, Wang Y, Rehman R, et al. STAT6 mediates the effect of ethanol on neuroinflammatory response in TBI. Brain Behav. Immun. 2019;81:228-46.

25. Wang J, Hou Y, Zhang L, Liu M, Zhao J, Zhang Z, et al. Estrogen attenuates traumatic brain injury by inhibiting the activation of microglia and astrocytemediated neuroinflammatory responses. Mol. Neurobiol. 2021;58:1052-61.

26. Dinet V, Petry KG, Badaut J. Brain-immune interactions and neuroinflammation after traumatic brain injury. Front. Neurosci. 2019;13:1178.

27. Mortezaee K, Khanlarkhani N, Beyer C, Zendedel A. Inflammasome: Its role in traumatic brain and spinal cord injury. J. Cell Physiol. 2018;233:5160-9.

28. Helmy A, Guilfoyle MR, Carpenter KLH, Pickard JD, Menon DK, Hutchinson PJ. Recombinant human interleukin-1 receptor antagonist promotes M1 microglia biased cytokines and chemokines following human traumatic brain injury. J. Cereb. Blood Flow. Metab. 2016;36:1434-48.

29. Kang TB, Yang SH, Toth B, Kovalenko A, Wallach D. Caspase-8 blocks kinase RIPK3mediated activation of the NLRP3 inflammasome. Immunity. 2013;38:27-40.

30. Feng J, Li M, Wei Q, Li S, Song S, Hua Z. Unconjugated bilirubin induces pyroptosis in cultured rat cortical astrocytes. J. Neuroinflammation. 2018;15:23.

31. McKenzie BA, Mamik MK, Saito LB, Boghozian R, Monaco MC, Major EO, et al. Caspase-1 inhibition prevents glial inflammasome activation and pyroptosis in models of multiple sclerosis. Proc. Natl Acad. Sci. USA. 2018;115:E6065-E6074.

32. Phillips FC, Gurung $P$, Kanneganti TD. Microbiota and caspase-1/caspase- 8 reg ulate IL-1 $\beta$-mediated bone disease. Gut Microbes. 2016;7:334-441.

33. Kang S, Fernandes-Alnemri T, Rogers C, Mayes L, Wang Y, Dillon C, et al. Caspase8 scaffolding function and MLKL regulate NLRP3 inflammasome activation downstream of TLR3. Nat Commun. 2015;6:7515.

34. Yamaguchi $A$, Ishikawa $H$, Furuoka $M$, Yokozeki $M$, Matsuda $N$, Tanimura $S$, et al. Cleaved PGAM5 is released from mitochondria depending on proteasomemediated rupture of the outer mitochondrial membrane during mitophagy. J Biochem. 2019;165:19-25.

35. Smith ED, Prieto GA, Tong L, Sears-Kraxberger I, Rice JD, Steward O, et al. Rapamycin and interleukin-1beta impair brain-derived neurotrophic factor-dependent neuron survival by modulating autophagy. J Biol Chem. 2014;289:20615-29.

36. Allan SM, Parker LC, Collins B, Davies R, Luheshi GN, Rothwell NJ. Cortical cell death induced by IL-1 is mediated via actions in the hypothalamus of the rat. Proc Natl Acad Sci USA. 2000:97:5580-5.
37. Cai Z, Lin S, Pang Y, Rhodes PG. Brain injury induced by intracerebral injection of interleukin-1 beta and tumor necrosis factor-alpha in the neonatal rat. Pediatr Res. 2004;56:377-84.

38. Fan LW, Tien LT, Zheng B, Pang Y, Rhodes PG, Cai Z. Interleukin-1beta-induced brain injury and neurobehavioral dysfunctions in juvenile rats can be attenuated by alpha-phenyl-n-tert-butyl-nitrone. Neuroscience. 2010;168:240-52.

\section{ACKNOWLEDGEMENTS}

This study was supported by the National Natural Science Foundation of China (No. 81901270, No. 82072229).

\section{AUTHOR CONTRIBUTIONS}

YHC, WL, and ZXW contributed to conceptualization. YHC and KG contributed to data analysis and graphing. LMG and BCZ performed in vitro experiments, and LMG contributed to RNA-seq data analysis and graphing. KG, BCZ, SFC and ZYL performed animal experiments. YHC contributed to the writing-original draft. WL and ZXW contributed to supervision, and $\mathrm{QHX}, \mathrm{WL}$, and ZXW contributed to writing review and editing.

\section{COMPETING INTERESTS}

The authors declare no competing interests.

\section{ADDITIONAL INFORMATION}

Supplementary information The online version contains supplementary material available at https://doi.org/10.1038/s41420-021-00686-8.

Correspondence and requests for materials should be addressed to Wei Liu or Zhanxiang Wang.

Reprints and permission information is available at http://www.nature.com/ reprints

Publisher's note Springer Nature remains neutral with regard to jurisdictional claims in published maps and institutional affiliations.

\begin{abstract}
c) Open Access This article is licensed under a Creative Commons Attribution 4.0 International License, which permits use, sharing, adaptation, distribution and reproduction in any medium or format, as long as you give appropriate credit to the original author(s) and the source, provide a link to the Creative Commons license, and indicate if changes were made. The images or other third party material in this article are included in the article's Creative Commons license, unless indicated otherwise in a credit line to the material. If material is not included in the article's Creative Commons license and your intended use is not permitted by statutory regulation or exceeds the permitted use, you will need to obtain permission directly from the copyright holder. To view a copy of this license, visit http://creativecommons. org/licenses/by/4.0/.
\end{abstract}

C The Author(s) 2021 